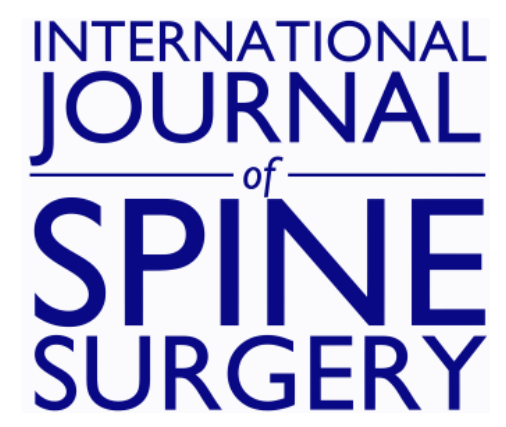

Pre-Operative Autologous Blood Donation Does Not Affect Pre-Incision Hematocrit in Adolescent Idiopathic Scoliosis Patients. A Retrospective Cohort of a Prospective Randomized Trial

Anthony J. Boniello, Kushagra Verma, Austin Peters, Baron S. Lonner and Thomas Errico

Int J Spine Surg 2016, 10 ()

doi: https://doi.org/10.14444/3027

http://ijssurgery.com/content/10/27

This information is current as of April 26, 2023.

Email Alerts Receive free email-alerts when new articles cite this article. Sign up at: http://ijssurgery.com/alerts

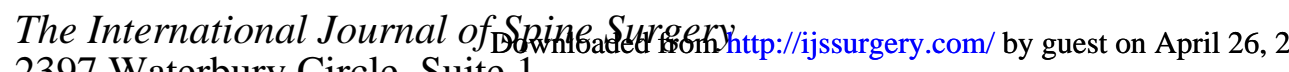
2397 Waterbury Circle, Suite 1, 


\section{Pre-Operative Autologous Blood Donation Does Not Affect Pre-Incision Hematocrit in Adolescent Idiopathic Scoliosis Patients. A Retrospective Cohort of a Prospective Randomized Trial}

Anthony J. Boniello, MD, 1,2 Kushagra Verma, $M S, M D,{ }^{1,3}$ Austin Peters, MD, ${ }^{1}$ Baron S. Lonner, MD, ${ }^{4}$ Thomas Errico, $M D^{1}$

${ }^{1}$ Division of Spinal Surgery, Department of Orthopaedic Surgery, NYU Hospital for Joint Diseases, New York, NY ${ }^{2}$ Department of Orthopaedic Surgery, Drexel College of Medicine, Hahnemann University Hospital, Philadelphia, PA ${ }^{3}$ Department of Orthopaedic Surgery, University of California, San Francisco, San Francisco, CA ${ }^{4}$ Department of Orthopaedic Surgery, Mount Sinai Beth Israel, New York, New York

\section{Abstract}

\section{Background}

Pre-donation of autologous blood prior to spine fusion for adolescent idiopathic scoliosis (AIS) has been used in deformity surgery. The effect of pre-donation on pre-operative hematocrit (Hct) remains debated. Multiple factors may influence pre-operative Hct including intravascular volume status, patient factors, and timing of pre-operative blood donation. The purpose of this study was to determine if pre-donation significantly lowers pre-incision Hct in AIS patients.

\section{Methods}

A retrospective cohort study of a Level-1 prospective randomized trial was conducted. 125 patients from the homogeneous population were included. AIS patients undergoing a posterior only spinal fusion for AIS were separated into two groups based on their pre-operative blood donation history. Demographic variables, pre-incision Hct, and transfusion rates were compared between the two groups using the Student's T-test.

Results

Pre-donation and non pre-donation groups had 28 and 97 patients, respectively. Pre-donation group was 75\% female $(21 \mathrm{~F}, 7 \mathrm{M})$ and non pre-donation group was $78 \%$ female $(76 \mathrm{~F}, 21 \mathrm{M})$. There was no difference between predonation and non pre-donation groups in mean age $(15.6 \pm 2.2$ vs $14.8 \pm 2.2, \mathrm{p}=0.081)$, BMI $(23.1 \pm 4.2$ vs $21.7 \pm$ 5.3, $\mathrm{p}=0.219)$, and pre-incision Hct $(32.8 \pm 3.4$ vs $33.8 \pm 3.1, \mathrm{p}=0.628)$. The overall transfusion rates were equivalent $(32.1 \pm 48.0 \%$ vs $25.8 \pm 44.0 \%, \mathrm{p}=0.509)$, however, the rate of allogenic transfusion for the pre-donation group was significantly lower $(3.6 \pm 18.9 \%$ vs $25.8 \pm 44.0 \%$, $\mathrm{p}=0.011)$.

\section{Conclusions}

This study supports the use of pre-donation for AIS, without a significant drop in pre-incision Hct. Patients that donate are also much less likely to be exposed to allogenic blood. There may be a surgeon bias to recommend predonation in patients with a larger BMI and older age. Future studies are needed from a larger population of patients including those with non-AIS pathology. Level of evidence: Level III.

KEYWORDS: AUTOLOGOUS BLOOD, ADOLESCENT IDIOPATHIC SCOLIOSIS, SPINE SURGERY, BLOOD TRANSFUSION, HEMATOCRIT VOLUME 10 ARTICLE 27 DOI: $10.14444 / 3027$

\section{Introduction}

Pre-donation of autologous blood (PD) prior to spine fusion for adolescent idiopathic scoliosis (AIS) in conjunction with other blood conservation techniques have been frequently used in spinal deformity surgery. PD has been extensively studied in non- spinal orthopaedic procedures and found to lower the risk of allogenic blood transfusions in hip and knee replacement surgeries. ${ }^{1-3} \mathrm{PD}$ has become increasingly common for elective spine surgery patients and can lead to a decrease in the likelihood of homologous blood transfusion. ${ }^{4 \cdot 6}$ Despite this increasing use, there exists a paucity of research on its 
efficacy and indications, 7 particularly with AIS surgery. In addition, parents of AIS patients are especially interested in reducing the potential for allogenic blood transfusion. In recent years this has driven surgeons to have greater focus on minimizing this risk through a variety of techniques including reduction of mean arterial pressure, meticulous surgical technique in conjunction with reduced operative time, and utilization of antifibrinolytic medications.

Various viruses such as HIV and Hepatitis B and C progressively contaminated donated allogenic blood in the 1980s. Therefore, healthcare professions sought to decrease the risk of exposure to contaminated products and in the late 1980s and 1990s many surgeons used PD for surgical procedures with large volumes of expected blood loss, such as in spinal surgery. ${ }^{9.12}$ Due to prohibitive costs of PD programs and decreased exposure risks from implementation of screening protocols PD began to fall out of favor. Consequently, the practice of autologous blood transfusion for spine surgeries decreased in many practices. However many of these conclusions have been based on cost analyses studies from the 1990s."

Pediatric spinal surgery is associated with significant blood loss; reported to vary from 57 to $350 \mathrm{~mL} /$ level fused, depending on surgical technique and instrumentation utilized. ${ }^{13}$ Greater blood loss increases the necessity for transfusion, and consequently the exposure to more blood products with the potential for disease transmission or transfusion reactions and increased surgical site infections. ${ }^{14,15}$ Using the National Inpatient Database it is estimated that 2.71 million spinal fusions were performed in the United States from 2002 to $2009,{ }^{16}$ of which $30 \%$ received blood transfusions. ${ }^{4,17}$ Although serious complications of allogenic transfusions are uncommon, the high rate of blood transfusions necessitate investigating alternative options.

The effect of autologous donation on pre- and postoperative hematocrit (Hct), and transfusion rates remains debated in the setting of AIS fusion surgery. Universally accepted indications to pre-donate in spine patients do not exist, particularly with AIS patients. Multiple factors may influence preoperative Hct including intravascular volume status, patient factors, and timing of pre-operative donation. This study aims to determine if AIS patients that predonate autologous blood have a significantly lower pre-incision Hct, post surgical Hct, and transfusion rates compared to AIS patients that did not predonate blood.

\section{Materials and Methods}

\section{Population}

We retrospectively examined a cohort of AIS patients undergoing a posterior only spinal fusion from a prospective randomized trial (Figure 1). ${ }^{8}$ During the prospective trial 125 patients were randomized to Tranexamic acid (TXA), Aminocaproic acid (EACA), or Saline (control) for surgery from January 2009 to January 2011. Level-1 data from this homogeneous population minimizes the effect of confounding variables. For the current study the $125 \mathrm{pa}-$ tients were separated into two groups based on their pre-operative blood donation history and compared in terms of their pre-incision Hct. All patients in the prospective study had a complete blood count drawn just prior to incision. ${ }^{8}$ Of the 125 patients enrolled in that study: 28 patients donated blood prior to surgery (PD group), 97 patients did not pre-donate blood (Non-PD group).
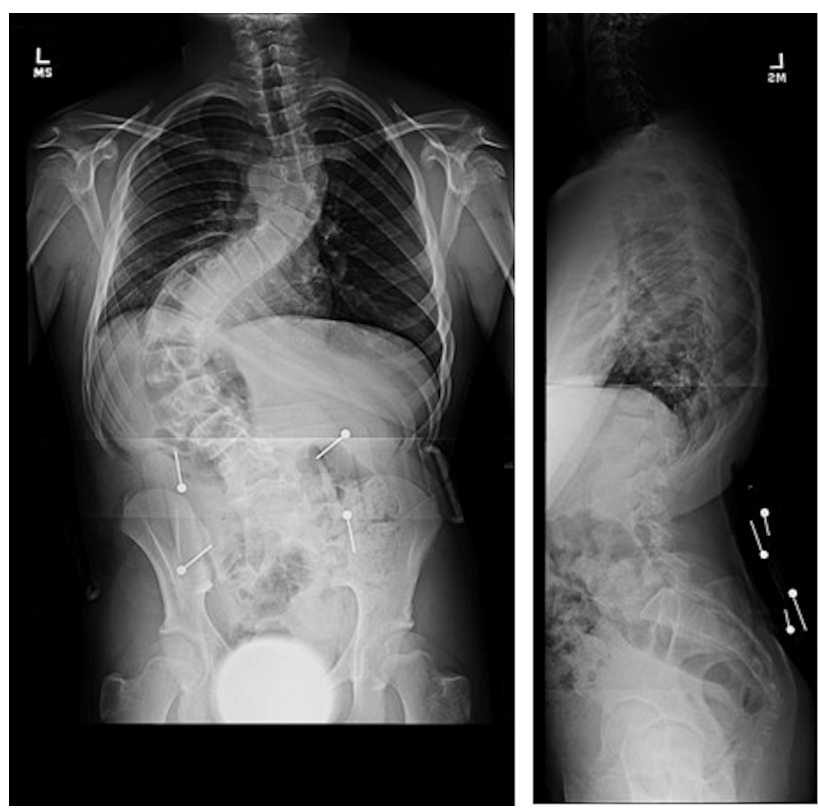

Fig. 1. Pre-operative coronal (left) and sagittal (right) radiographs of a 17 year old male with adolescent idiopathic scoliosis. Main Thoracic curve $=71$ degrees (bending= 54); TL/L= 91 degrees (bending= 73). 
Pre-donation program

There is no age limitation to pre-donate blood at the study institution. Absolute exclusion criteria for predonations include: active infection and weight less than $100 \mathrm{lbs}$. Relative exclusion criteria include: cardiac conditions, pulmonary disease, current anticoagulant therapy, and a history of cerebrovascular disease, or seizures. Relative exclusion criteria are considered on a case-by-case basis. Patients may donate blood every four to seven days, and up to three days before surgery with up to two units donated in the month prior to surgery. Patients are instructed to avoid acetaminophen, aspirin and alcohol consumption for 48 hours prior to donation.

\section{Surgery}

Posterior spinal fusion was achieved by careful dissection of the paraspinal musculature from the spinous process, lamina, and facet. Soft tissue releases and ponte osteotomies on the concavity were performed to increase flexibility followed by pedicle screw fixation. (Figure 1 and Figure 2) Local autologous and allograft bone graft was used to achieve bony fusion. Sub-fascial hemovac drains were routinely placed at the wound site during closure and uniformly removed post-operatively when wound drainage was less than $40 \mathrm{ml}$ per eight-hour shift. Transfusion was performed intraoperatively if a patient had a measured Hct of less than 25 with ongoing bleeding, and post-operatively if a symptomatic patient had a Hct of less than 22 .

\section{Statistical Methods}

Demographic variables analyzed include age, BMI, gender, and estimated blood volume. Estimated blood volume was approximated as $80 \mathrm{~mL} / \mathrm{kg}{ }^{18}$ The primary outcome measure was pre-incisional Hct. To insure the proportion of patients assigned to EACA, TXA and/or saline did not differ between PD and non-PD groups a Pearson chi-squared test was performed. Additional outcome measures analyzed include peri-operative transfusion rate, length of hospitalization, estimated blood loss (EBL), and Hct on post-operative day (POD) 1 and 2. The effects of Tranexamic acid (TXA), Aminocaproic acid (EA$\mathrm{CA}$ ), and Saline (control) were not analyzed as the primary outcome was pre-incisional Hct, and there were too few subjects to do a sub-analyses on sec- ondary outcome measures. Outcome variables were statistically analyzed using SPSS software (SPSS, Inc.). Demographic variables and study outcomes were compared between the two groups using the Student's T-test with statistical significance was defined as $\mathrm{p}<0.05$.

\section{Results}

\section{Demographics}

The proportion of patients assigned to EACA, TXA and/or saline did not differ between PD and non-PD groups $(\mathrm{p}=0.26)$. The $\mathrm{PD}$ group was $75 \%$ female $(21 \mathrm{~F}, 7 \mathrm{M})$, and Non-PD group was $78 \%$ female $(76 \mathrm{~F}$, $21 \mathrm{M})$. The mean age of the PD group trends to a higher age $(15.6 \pm 2.2$ vs $14.8 \pm 2.2$ years, $\mathrm{p}=0.081)$, although this was not statistically significant. The BMI of the PD was slightly higher than Non-PD ( $23.1 \pm 4.2$ versus $21.7 \pm 5.3, \mathrm{p}=0.219)$, although this difference was not statistically significant. The estimated blood volume for the patients that predonated was significantly higher $(4444 \pm 1142$ vs 3881 $\pm 1060, \mathrm{p}=0.017)$. (Table 1)
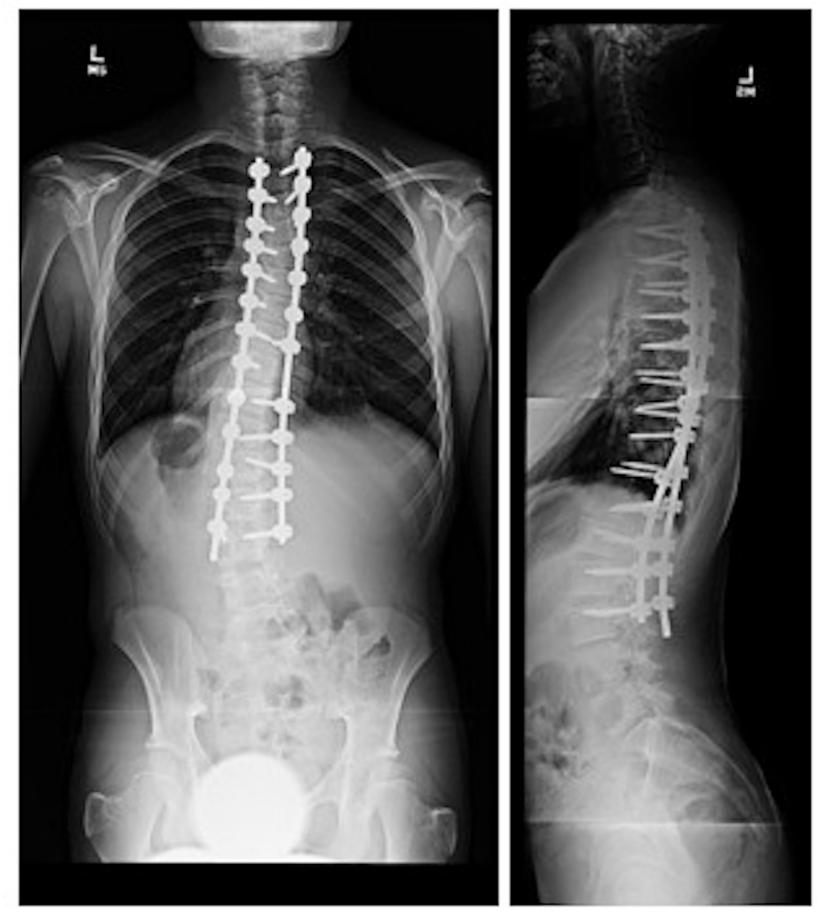

Fig. 2. Post-operative coronal (left) and sagittal (right) radiographs of a 17 year old male with adolescent idiopathic scoliosis who underwent posterior spinal fusion (T3-L3). Estimated blood loss was $4500 \mathrm{cc}$ and 3 units of allogenic packed red blood cells were transfused. 
Hematocrit

Hct between the two groups (PD vs Non-PD) were equivalent at the three time points measured: The pre-incision Hct $(32.8 \pm 3.4$ vs $33.8 \pm 3.1, \mathrm{p}=0.167)$, POD1 Hct $(29.3 \pm 4.7$ vs $28.5 \pm 3.4, \mathrm{p}=0.333)$, POD2 Hct $(28.2 \pm 4.4$ vs $27.3 \pm 3.6, \mathrm{p}=0.309)$, and change in Hct from pre-incision to POD2 $(-3.6 \pm 3.6$ vs $-5.3 \pm$ $5.9, \mathrm{p}=0.154)$ did not differ significantly (Table 2 ).

\section{Surgical Parameters}

The overall transfusion rate between the PD and Non-PD did not differ significantly $(32.1 \pm 48.0 \%$ vs $25.8 \pm 44.0 \%, \mathrm{p}=0.509)$. However, the rate of allogenic transfusion for the PD group was significantly lower than the Non-PD group $(3.6 \pm 18.9 \%$ vs $25.8 \pm$ $44.0 \%, \mathrm{p}=0.011)$. Length of hospitalization did not differ significantly between PD and Non-PD (5.2 \pm 0.8 days vs $5.5 \pm 1.1, \mathrm{p}=0.225)$. Therefore, patients who pre-donate blood are less likely to be exposed to allogenic blood, without increasing the risk of overall transfusion and without an increased length of hospitalization (Table 3).

\section{Estimated Blood Volume}

Patients who pre-donated blood trended towards losing less total blood intra-operatively $(734 \pm 734 \mathrm{cc}$ vs $938 \pm 742 \mathrm{cc}, \mathrm{p}=0.180)$, and less blood loss per level fused $(77.8 \pm 62.1 \mathrm{cc}$ vs $102.5 \pm 65.7 \mathrm{cc}, \mathrm{p}=0.079)$, although these trends were not statistically significant. Interestingly, patients that pre-donated lost a significantly smaller portion of their total estimated blood

Table 1. Demographic information. (PD = Pre-donate of autologous blood, Non-PD = No pre-donation of autologous blood).

\begin{tabular}{|l|r|r|r|r|}
\hline Group & N & Mean Age & BMI & Gender (\%female) \\
\hline PD & 28 & $15.6 \pm 2.2$ & $23.1 \pm 4.2$ & 75 \\
\hline NON-PD & 97 & $14.8 \pm 2.2$ & $21.7 \pm 5.3$ & 78 \\
\hline p & - & 0.081 & 0.219 & - \\
\hline
\end{tabular}

Table 2. Hematocrit ( $\mathrm{Hct}$ ) measured at pre-incision, post-operative day (POD) 1, POD2, and change from pre-incision to POD2. (PD = Pre-donate of autologous blood, Non-PD = No pre-donation of autologous blood).

\begin{tabular}{|l|r|r|r|r|}
\hline Group & $\begin{array}{r}\text { Pre-Incision } \\
\text { Het }\end{array}$ & $\begin{array}{r}\text { POD1 } \\
\text { Het }\end{array}$ & $\begin{array}{r}\text { POD2 } \\
\text { Het }\end{array}$ & $\begin{array}{r}\text { Change in Het (In- } \\
\text { cision to POD2) }\end{array}$ \\
\hline PD & $32.8 \pm 3.4$ & $29.3 \pm 4.7$ & $28.2 \pm 4.4$ & $-3.6 \pm 3.6$ \\
\hline $\begin{array}{l}\text { NON- } \\
\text { PD }\end{array}$ & $33.8 \pm 3.1$ & $28.5 \pm 3.4$ & $27.3 \pm 3.6$ & $-5.3 \pm 5.9$ \\
\hline p & 0.167 & 0.333 & 0.309 & 0.154 \\
\hline
\end{tabular}

volume $(17 \pm 13 \%$ vs $25 \pm 19 \%$, p=0.012). (Table 4$)$

\section{Discussion}

Pre-donation prior to surgical procedures has the potential to minimize the risks of allogenic blood transfusions. ${ }^{4}$ Allogenic transfusion is associated with potentially serious complications such as; incorrect blood component transfused (CMV + blood to a CMV- patient), acute transfusion reactions, as well as transfusion-related acute lung injury. ${ }^{19}$ Allogenic transfusion also increase the risk of infectious disease such as hepatitis B and C, HIV and nvCJD. ${ }^{19}$ Predonation has several limitation in major spinal surgery such as difficulty of predicting which patients require blood tranfusion, ${ }^{20,21}$ the high potential for wastage (up to $40 \%$ discarded autologous blood products) ${ }^{20}$ a high level of communication across a variety of specialties necessary for successful implementation, and its inavailability in emergency surgeries. $^{4}$

With the exception of the estimated blood volume, the baseline demographic variables between the PD and non-PD groups were similar. Due to institutional policy, patients were excluded from pre-donation if they weighed less than 100 lbs. The significantly larger estimated blood volume in the pre-donation group is therefore not surprising. Similarly, this exclusion criterion likely explains the trend towards larger BMI

\begin{tabular}{|c|c|c|c|}
\hline Group & $\begin{array}{r}\text { Transfusion } \\
\text { Rate (\%) }\end{array}$ & $\begin{array}{r}\text { Allogenic Blood } \\
\text { Transfusion Rate (\%) }\end{array}$ & LOH (days) \\
\hline PD & $32.1 \pm 48.0$ & $3.6 \pm 18.9$ & $5.2 \pm 0.8$ \\
\hline NON-PD & $25.8 \pm 44.0$ & $25.8 \pm 44.0$ & $5.5 \pm 1.1$ \\
\hline $\mathbf{p}$ & 0.509 & 0.011 & 0.225 \\
\hline
\end{tabular}

Table 4. Estimated Blood Loss. Bolded p-value denotes statistical significance. $(P D=$ Pre-donate of autologous blood, Non-PD = No pre-donation of autologous blood, EBL = Estimated Blood Loss, EBV = Estimated Blood Volume).

\begin{tabular}{|l|r|r|r|r|}
\hline Group & EBL (mL) & EBV (mL) & EBL/Levels fused & EBL/EBV \\
\hline PD & $734 \pm 734$ & $4444 \pm 1142$ & $77.8 \pm 62.1$ & $0.17 \pm 0.13$ \\
\hline Non-PD & $938 \pm 742$ & $3881 \pm 1060$ & $102.5 \pm 65.7$ & $0.25 \pm 0.19$ \\
\hline $\mathbf{p}$ & 0.180 & $\mathbf{0 . 0 1 7}$ & 0.079 & $\mathbf{0 . 0 1 2}$ \\
\hline
\end{tabular}

Downloaded from http://ijssurgery.com/ by guest on April 26, 2023 
and age in the PD group. This data suggests that PD patients were comparable to Non-PD.

One study by Brookfield et al. has found the greatest drawback to the use of PD in major spine surgery is that it lowers pre-operative hemoglobin level. ${ }^{20}$ Similarly, Kennedy et al. examined elective posterior lumbar spine surgery in adult patients and found PD induced preoperative anemia. The authors found preoperative Hct in patients who pre-donated was significantly lower $(39.8 \pm 4.5$ vs $41.6 \pm 4.6, \mathrm{p}<0.001)$. The PD group in the present study had an equivalent pre-incision Hct as compared to the Non-PD group. The predonation program, and mean time from predonation was unfortunately not available to compare among the present study, and Kennedy et al. and Brookfield et al. While our study shows a contrasting result, it is important to recognize that AIS patients are a generally healthy uniform population that is likely better able to compensate for the blood loss necessary for predonation. Perhaps these contrasting findings represent an inherent difference in the populations analyzed. Kennedy et al. and Brookfield et al. studied older populations. Therefore, the results of this study are best applied to a healthy population of adolescents or young adults.

In the setting of posterior lumbar surgery Kennedy et al. found a lower transfusion threshold in PD than allogenic blood, likely due to the higher rate of preoperative anemia in the studied patient population or the belief that potential infectious complications from contaminated blood would be negligibile. ${ }^{7}$ This study found that PD group patients who donated one and two units of blood were, respectively, 7.5 and 9 times more likely to be transfused within the first 24 hours than Non-PD patients. ' Conversely, a retrospective study by Solves et al. of patients undergoing major elective spine surgery found that pre-donation was effective for reducing allogeneic transfusion rates in mostly young patients undergoing major elective spinal surgery (Odds Ratio $=0.077 ; 95 \% \mathrm{CI}=$ $0.043-0.140) .{ }^{6}$ The present study found that predonation did not increase the risk of transfusion in the peri-operative period. The drastic reduction in the risk of allogenic transfusion seen as well is a result of the use of autogenic prior to the use of allogenic blood. These findings coincide with the find- ings by Solves et al. ${ }^{6}$ This reduced rate of allogenic transfusion in patients who pre-donate, with no increased risk of overall transfusion risk effectively reduces the potential for disease transmission or transfusion reactions. ${ }^{14}$

The present study did not investigate the effects of instrumentation on transfusion rates or Hct, however Kennedy et al. found that pre-donation was significantly more efficient in patients who underwent instrumented lumbar fusion than in patients with less complex surgery. ${ }^{7}$ Cha et al. found that pre-donation decreased their risk of receiving allogeneic blood by $75 \%$ for non-instrumented fusions, and $50 \%$ for instrumented fusions in posterior lumbar decompression with fusion of up to three levels. ${ }^{21}$ This study concluded PD significantly diminishes allogeneic blood usage in patients undergoing spinal fusion. ${ }^{21}$

This study is limited by its small sample size and inclusion of relatively few surgical and patient factors likely to influence blood loss including timing of predonation, and curve type. Furthermore, the difference in BMI between the two groups suggests there may be a surgeon bias to recommend pre-donation in patients with a larger BMI, although this difference was not significant. This bias may be a function of longer surgery times required for patients with larger BMIs. Additionally the study did not track peri- and post-operative complications, and therefore definitive conclusions concerning reducing the rate of transfusion reactions and exposure to infectious diseases cannot be made.

Another limitation of the current study is costanalysis. Unfortunately the cost incurred for one unit of PD was not available at the studied institution. The cost effectiveness of pre-donation has been previously discussed, however much of the literature that may have contributed to the decreased use of PD are cost analyses studies from the 1990s and early 2000 s. $^{11,21}$ For example, in 2002 Cha et al. concluded routine preoperative collection of autologous blood is not necessary before simple lumbar laminectomy without fusion despite reducing allogenic transfusion rate in lumbar laminectomy procedures because the donated blood is discarded in $80 \%$ of cases. ${ }^{21}$ Since Medicare does not reimburse for pre-donation ${ }^{22}$ the 
cost of the surgery must be covered by insurance, or more frequently by the patient. One 2003 study analyzed the use of autologous pre-donated blood transfusion in surgery for scoliosis in 45 patients at one hospital in England. ${ }^{23}$ The authors concluded that autologous blood transfusion required extra time, personnel, resources and cost $£ 28.88$ ( $\$ 47.15$ USD) per patient more than allogenic transfusion. ${ }^{23}$ Additional concerns about unused autologous blood adding an unnecessary expense has been raised as well. ${ }^{2,24}$ To date the cost of a unit of allogenic blood is variable and institution dependent. The cost is also increased by the various tests and processing necessary for minimize patient risk.

More recent literature supports the use of $\mathrm{PD}$ as a cost-effective method. In 2004 Hardwhick et al. concluded PD to be similar in cost to allogenic blood in orthopaedic procedures, specifically in total hip replacement procedures. This study found the mean cost of one unit of PD to be $\$ 391$ and one unit of allogeneic blood to be $\$ 514$. $^{25}$ These findings highlight the need for further research on the costeffectiveness of PD programs, particularly in the AIS population.

This study's strength is its inclusion of a homogenous population of AIS patients participating in a level-one study with a very specific transfusion trigger. Future studies are needed from a larger population of patients, including those with non-AIS pathology, to make more definitive suggestions on optimal protocol (timing, frequency and indications). Future studies will also be necessary to develop guidelines based on volume status, curve type and magnitude, planned procedure, and BMI. Queries of multi-center databases may offer additional supportive evidence.

Based on the findings of the current study, surgeons can consider pre-operative donation of autologous blood in patients undergoing planned AIS spine surgery without fear of significantly decreasing the patient's Hct at incision, or increasing the rate of overall transfusions. The decreased rate of allogenic transfusions would make this a safe and effective alternative in patients that meet inclusion criteria for pre-donation programs. However the potential risks of allogenic or autologous blood transfusion, benefits and cost of PD must be thoroughly discussed with the patient prior to its utilization.

\section{References}

1. Bou Monsef J, Buckup J, Mayman D, Marx R, Ranawat A, Boettner F. Targeted preoperative autologous blood donation in total knee arthroplasty reduces the need for postoperative transfusion. HSS J. 2013;9(3):214-217. doi:10.1007/s11420-013-9346-8. 2. Oberhofer D, Sakić K, Janković S, Tonković D, Vrgoc G. [How to improve perioperative blood management in patients undergoing total hip or knee re-

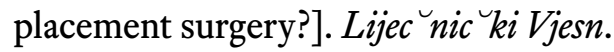
134(11-12):322-327. http://www.ncbi.nlm.nih.gov/ pubmed/23401978. Accessed January 20, 2016. 3. Billote DB, Glisson SN, Green D, Wixson RL. Efficacy of preoperative autologous blood donation: analysis of blood loss and transfusion practice in total hip replacement. J Clin Anesth. 2000;12(7):537-542. doi:10.1016/S0952-8180(00)00213-0.

4. Kumar N, Chen Y, Nath C, Liu EHC. What is the role of autologous blood transfusion in major spine surgery? Am J Orthop (Belle Mead NJ). 2012;41(6):E89-E95.

5. Goodnough L, Marcus R. Effect of autologous blood donation in patients undergoing elective spine surgery. Spine (Phila Pa 1976). 1992.

6. Solves P, Carpio N, Moscardo F, et al. Results of a preoperative autologous blood donation program for patients undergoing elective major spine surgery. Transfus Apher Sci. July 2013:4-7. doi:10.1016/ j.transci.2013.06.016.

7. Kennedy C, Leonard M, Devitt A, Girardi FP, Cammisa FP. Efficacy of preoperative autologous blood donation for elective posterior lumbar spinal surgery. Spine (Phila Pa 1976). 2011;36(26):E1736-E1743. doi:10.1097/ BRS.0b013e3182194a42.

8. Verma K, Errico TJ, Vaz KM, Lonner BS. A prospective, randomized, double-blinded single-site control study comparing blood loss prevention of tranexamic acid (TXA) to epsilon aminocaproic acid (EACA) for corrective spinal surgery. BMC Surg. 2010;10:13. doi:10.1186/1471-2482-10-13.

9. Goodnough LT, Brecher ME. Autologous blood 
transfusion. Intern Med. 1998;37(3):238-245.

10. Bernstein LH, Coles M, Viner N. Bridgeport Hospital autologous blood donation experience from 1992 to 1996. Yale J Biol Med. 1996;68(5-6):207-213. http://www.pubmedcentral.nih.gov/articlerender.fcgi? artid $=2588941 \&$ tool $=$ p....

11. Roberts W a, Kirkley S a, Newby M. A cost comparison of allogeneic and preoperatively or intraoperatively donated autologous blood. Anesth Analg. 1996;83(1):129-133. http://www.ncbi.nlm.nih.gov/ pubmed/8659723.

12. Tulloh BR, Brakespear CP, Bates SC, et al. Autologous predonation, haemodilution and intraoperative blood salvage in elective abdominal aortic aneurysm repair. Br J Surg. 1993;80(3):313-315. 13. Shapiro F, Sethna N. Blood loss in pediatric spine surgery. Eur Spine J. 2004;13 Suppl 1:S6-S17. doi:10.1007/s00586-004-0760-y.

14. Hu SS. Blood loss in adult spinal surgery. Eur Spine J. 2004;13 Suppl 1:S3-S5. doi:10.1007/ s00586-004-0753-x.

15. Schwarzkopf R, Chung C, Park JJ, Walsh M, Spivak JM, Steiger D. Effects of perioperative blood product use on surgical site infection following thoracic and lumbar spinal surgery. Spine (Phila $\mathrm{Pa}$ 1976). 2010;35(3):340-346. doi:10.1097/

BRS.0b013e3181b86eda.

16. Nandyala S V, Marquez-Lara A, Fineberg SJ, Singh R, Singh K. Incidence and risk factors for perioperative visual loss after spinal fusion. Spine J. November 2013. doi:10.1016/j.spinee.2013.10.026.

17. Berenholtz SM, Pronovost PJ, Mullany D, et al. Predictors of transfusion for spinal surgery in Maryland, 1997 to 2000. Transfusion. 2002;42(2):183-189. 18. Yamamoto LG. Case Based Pediatrics for Medical Students and Residents. AuthorHouse; 2004.

19. Harrison E, Bolton P. Serious hazards of transfusion in children (SHOT). Paediatr Anaesth.

2011;21(1):10-13. doi:10.1111/

j.1460-9592.2010.03474.x.

20. Brookfield KF, Brown MD, Henriques SM, Buttacavoli FA, Seitz AP. Allogeneic transfusion after predonation of blood for elective spine surgery. Clin Orthop Relat Res. 2008;466(8):1949-1953. doi:10.1007/s11999-008-0306-4.

21. Cha CW, Deible C, Muzzonigro T, Lopez-Plaza I, Vogt M, Kang JD. Allogeneic transfusion requirements after autologous donations in posterior lumbar surgeries. Spine (Phila Pa 1976). 2002;27(1):99-104.

22. American Medical Association. $C P T^{\circledR} 2014$ Professional Edition: Spiralbound. American Medical Association; 2014.

23. Ridgeway S, Tai C, Alton P, Barnardo P, Harrison DJ. Pre-donated autologous blood transfusion in scoliosis surgery. J Bone Jt Surg.

2003;85(7):1032-1036. doi:10.1302/

0301-620X.85B7.13466.

24. Lenke LGBKHS-MKHM. Wasting of preoperatively donated autologous blood in the surgical treatment of adolescent idiopathic scoliosis. Spine (Phila Pa 1976). 2006;31(20):2375-2380.

25. Hardwick ME, Morris BM, Colwell CW. Twodose Epoetin Alfa Reduces Blood Transfusions Compared with Autologous Donation. Clin Orthop Relat Res. 2004;423(423):240-244. doi:10.1097/ 01.blo.0000130206.58617.9e.

\section{Disclosures \& COI}

Anthony Boniello reports no relevant disclosures or COI; Thomas Errico reports no relevant disclosures or COI; Baron Lonner reports no relevant disclosures or COI; Austin Peters reports no relevant disclosures or COI; Kushagra Verma reports no relevant disclosures or COI.

\section{Corresponding Author}

Anthony Boniello, Department of Orthopedic Surgery, Drexel College of Medicine, Hahnemann University Hospital, Philadelphia, PA. anthony.boniello@gmail.com

Published 16 August 2016.

This manuscript is generously published free of charge by ISASS, the International Society for the Advancement of Spine Surgery. Copyright @ 2016 ISASS. To see more or order reprints or permissions, see http://ijssurgery.com. 